\title{
Satisfaction and Knowledge of Elderly Patients Towards Their Multiple Medications Usage
}

\author{
SM Alqallaf* and Z Ali \\ Allied Health Sciences Department, University of Bahrain, Bahrain
}

*Corresponding author: Pharmacy program, Allied Health Sciences Department, College of Health \& Sports Sciences, University of Bahrain, Bahrain.

\begin{abstract}
Multiple drug use is considered to be hazardous generally and in specific for the elderly because of their greater vulnerability to complications. The aim of this study is to determine the satisfaction and knowledge of elderly patients in Bahrain towards their multiple medications usage and to discover the prevalence of inappropriate polypharmacy among elderly patients in Bahrain. In a cross-sectional study, 100 participants age more than 55 years were interviewed to determine the prevalence of polypharmacy. The participants were randomly selected and asked to answer a questionnaire. The questionnaire consisted of questions regarding drugs taken, pattern of using each drug and patients' personal, social and medical history in addition to their satisfaction with their treatment regimen. The data collected were analyzed using Microsoft Excel. Participants were found to consume an average of $8.14 \pm 1.22$ of drugs and the average number of tablets / capsules were $9.88 \pm 1.21$. The most commonly used drugs were aspirin, atorvastatin, metformin, and beta-blockers. Those were prescribed by physicians in $82 \%$ of cases. Participants using herbal products / nutritional supplements were found to be $34 \%$. The desire of participants to reduce the number of their medications was found to be high (83\%) as many believed that at least one of them is causing side effects. Polypharmacy is common among elderly population in Bahrain and is affected by age, education level and medical factors. Different measures need to be implemented to minimize this problem to ensure better health for the elderly population.
\end{abstract}

Keywords: Polypharmacy; Multiple medications; Satisfaction

\section{Background and Aims}

Although multiple drug use or polypharmacy might be considered appropriate as in cases of concomitant diseases or in complex medical conditions, the inappropriate polypharmacy or major polypharmacy is a very common problem around the globe [1-4]. An example of that is a German study which reported the use of more than 5 medications in $26.7 \%$ of elderly patients and this doubles (54\%) when including over the counter medications [1]. Nevertheless, the number of medications that constitutes polypharmacy is not fully agreed. However, WHO (2004) defined Polypharmacy as "the administration of many drugs at the same time or the administration of an excessive number of drugs "WHO 2004. Hence, polypharmacy term has been used to describe a patient's use of multiple drugs and is used also more generally to describe unnecessary drug prescription $[3,5]$.

The main reason for the problem of inappropriate polypharmacy is the growing elderly population with its accompanying pathophysiological changes and the advancement in healthcare services [6-8]. Age-related changes involves all body organs / systems and necessitates special considerations in drug prescribing in the elderly [9]. These changes might affect drugs' pharmacokinetics parameters and might result in potential drugdrug interactions $[9,10]$. Other causes for the polypharmacy include visiting multiple physicians, the use of complex drug treatment and the occurrence of drugs' side effects which might be interpreted as 
a newly existing disease $[6,11,12]$. Another genuine reason is the psychosocial factor such as the use of traditional remedies or herbs which was reported in $30 \%$ of German adults $[6,8]$.

The evidence for the negative impact of polypharmacy on the patients' health is well established, even with the intake of as few as four medications at any given time [5,13]. One main complication is the exposure to potentially inappropriate medications with what it carries of an increase in the incidence of serious adverse drug reactions and interactions [2,6,13-16]. In Sweden, fatal adverse drug reactions represented approximately $3 \%$ of all deaths in elderly patients [15]. Non-fatal adverse drug reactions secondary to polypharmacy represents a significant cause of emergency department visits and hospital admission in the elderly [7,14-16]. A study indicated that $12 \%$ of hospital admissions in elderly are related to adverse drug reactions [14]. These patients were found to be at increased risk of rehospitalization [17]. Another complication of polypharmacy is the under-prescribing of recommended drugs; which is known as "treatment risk paradox" [6]. Polypharmacy is also associated with an increase in the risk of geriatric syndromes such as delirium, falls, incontinence and behavioral disturbances [14-16]. Other consequences of polypharmacy are higher mortality rate, greater health-care cost and increased medication errors [6, 14-18]. Additionally, multiple medication use carries the risk of poor adherence which is a multi-factorial problem [14]. Factors that might worsen the problem of poor adherence in patients with multiple medications include dosing schedule confusion, inadequate medication education / counselling, poor patient's memory, poor hand dexterity with difficult to open packages and it might intentional trying to avoid adverse drug reactions [6].

The aim of this study is to determine the satisfaction and knowledge of elderly patients in Bahrain towards their multiple medication's usage. Another aim is to discover the prevalence of inappropriate polypharmacy among elderly patients in Bahrain.

\section{Methods}

A systematic review of the literature was performed using several medical engines including the National Library of Medicine's PubMed database, Science Direct and Google Scholars (limited to English language). The terms used in the search were polypharmacy, multiple medication use, polypharmacy and elderly, inappropriate medication frequency, medication errors with polypharmacy. The search profile included comprehensive lists of various clinical studies that were conducted in different world areas for statistically relevant information about polypharmacy. Data of these studies were eventually compared with the analysis of the current study. A cross sectional survey was carried out aiming at exploring the prevalence of the problem of inappropriate polypharmacy. Using a standardized questionnaire, 100 Bahraini patients aged 55 to more than 75 years were interviewed in different. Because of the limited time available for completing the study being part of an educational course, convenience sampling was used and therefore the study may not be sufficiently representative of the entire population. The Inclusion criteria were patients aged more than 55 years. No exclusion criteria were put. Both genders were enrolled, in which fifty-five males and forty-five females were interviewed. Participants answered 14 questions regarding drugs taken, pattern of using each drug and patients' personal, social and medical history in addition to their satisfaction with their treatment regimen. The survey was pilot tested on 10 subjects to improve questions, clarity and quality of data collection. Full demographic data was collected for all subjects, including age, gender, and education Table 1.

A summary score of percentage correct answers was finally created. The data was analyzed using Microsoft Excel program for statistical analysis. Since the study is cross-sectional, descriptive statistical analysis was conducted. This study was approved by the Pharmacy Program Ethics Committees. Data was collected from participants who provided verbal consent.

\section{Results}

\section{Demographic data}

Demographical data of the participants are shown in Table 1. The male patients were $55 \%$, while the rest were female. The majority ( $49 \%$ ) of patients were 55 - 64 years age, $31 \%$ were 65 75 years and the rest (20\%) were more than 75 years. Illiterate patients were $15 \%$, patients with elementary, intermediate and secondary school certificates were 18\%, 16\% and $33 \%$ respectively, while patients with university degrees were $18 \%$. The results showed that the majority of participants suffered from diabetes (87\%), hypertension (84\%) and hyperlipidemia (83\%). Furthermore, some of the participants suffered from heart disease (38\%), thyroid gland disorders (32\%) and other chronic disease such as gout, glaucoma, depression, asthma and rheumatoid arthritis (Figure 1).

Table 1: Demographical data of the participants.

\begin{tabular}{|c|c|c|c|c|c|}
\hline \multirow{2}{*}{ Gender } & \multicolumn{2}{|c|}{ Female } & \multicolumn{3}{|c|}{ Male } \\
\hline & \multicolumn{2}{|c|}{$45 \%$} & \multicolumn{3}{|c|}{$55 \%$} \\
\hline \multirow{2}{*}{ Age Groups } & \multicolumn{2}{|c|}{$55-64$} & \multicolumn{2}{|c|}{$65-75$} & More than 75 \\
\hline & \multicolumn{2}{|c|}{$49 \%$} & \multicolumn{2}{|c|}{$31 \%$} & $20 \%$ \\
\hline \multirow{2}{*}{ Education } & Illiterate & Elementary & Intermediate & Secondary & University \\
\hline & $15 \%$ & $18 \%$ & $16 \%$ & $33 \%$ & $18 \%$ \\
\hline
\end{tabular}


The number of medications that patients take daily ranged from 4 to 26 with an average of $8.14 \pm 1.22$. The most commonly used drugs were acetylsalicylic acid, atorvastatin, metformin, atenolol and propranolol. These drugs were prescribed by physicians in over $82 \%$ of patients. The number of tablets or capsules used daily ranged from 6 to 27 with an average of $9.88 \pm 1.21$ (data not shown). It was found that $90 \%$ of participants felt that all their regular medications were necessary; while $5 \%$ felt that they were taking a medication they no longer need (Figure 2). The desire of the participants to reduce the number of medications they were taking currently was found to be high (83\%); while 3\% felt comfortable with the number of medications they were taking (Figure 3).

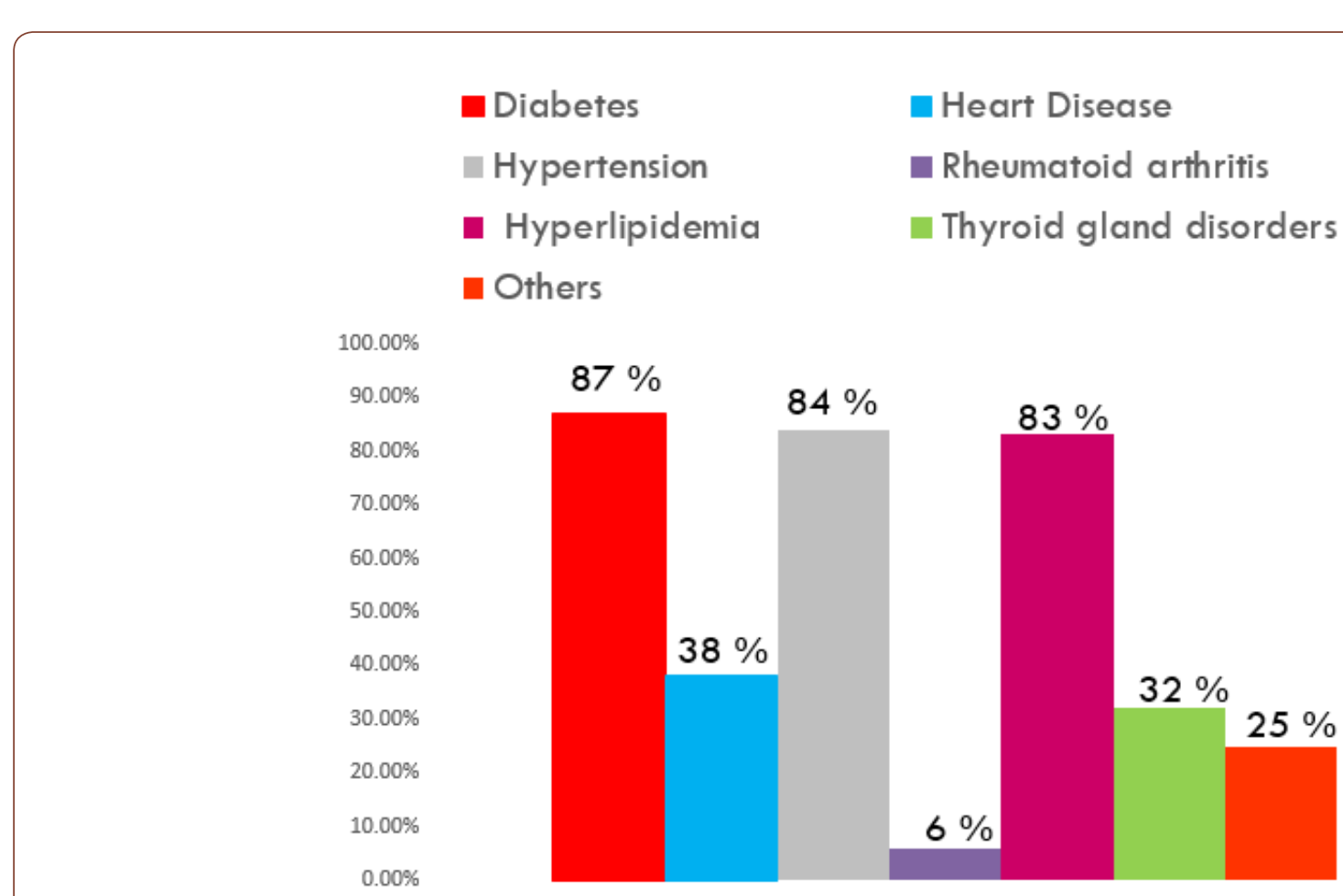

Figure 1: Health conditions that participants suffer from.

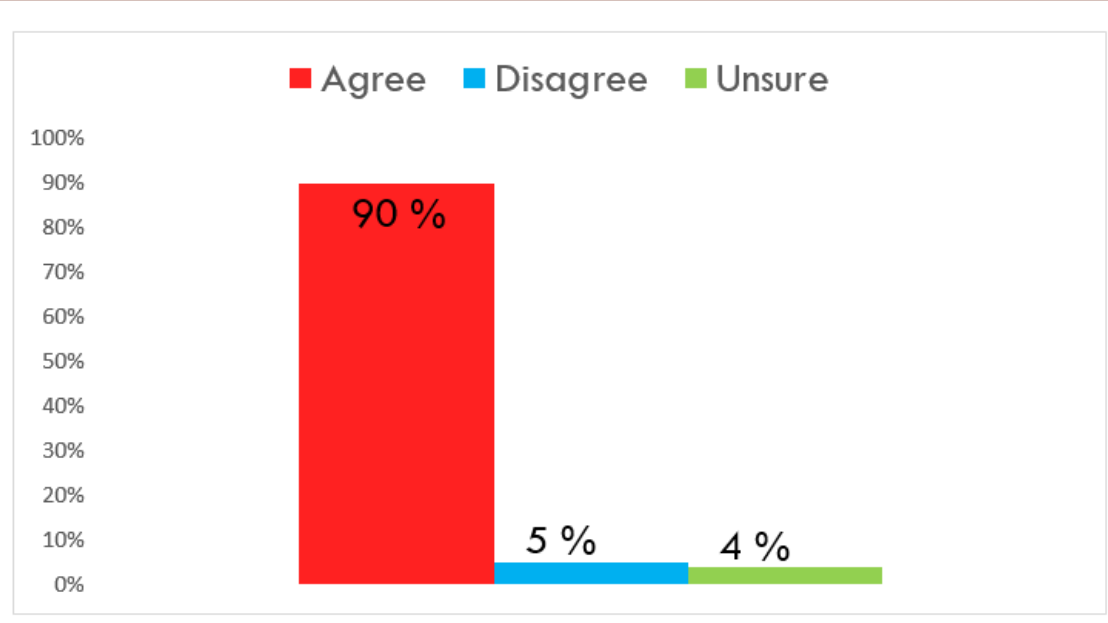

Figure 2: Participants belief of the necessity of their medications.

According to the results, $45 \%$ of the participants would accept taking more medications if their health condition necessitate; while $41 \%$ believed that what they were taking was enough (Figure 4 ).

The results showed that $88 \%$ of the participants had a good understanding of the reasons for prescribing each of their medications, but $2 \%$ had no idea (Figure 5). It was found that $53 \%$ of the participants suffered from adverse effect(s) from at least one of their medications, while only $7 \%$ stated that their medications didn't give them any side effects effects (Figure 6). 


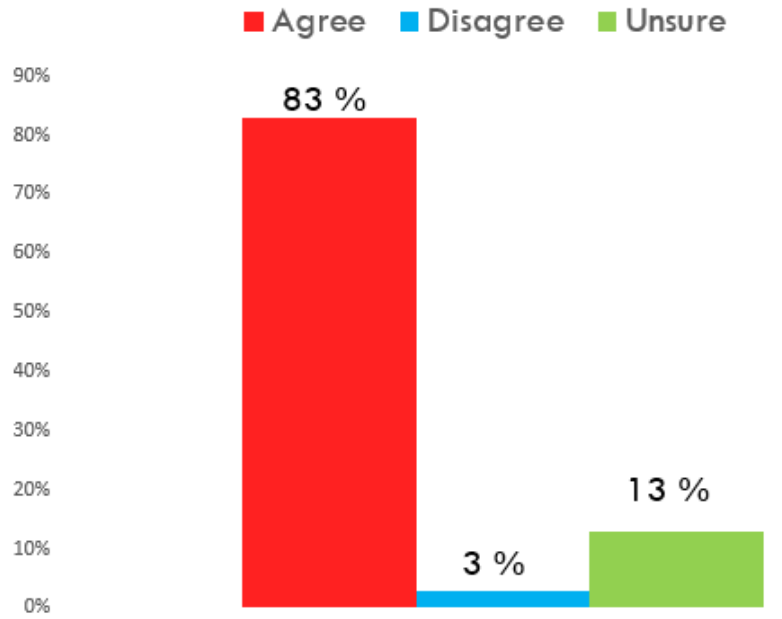

Figure 3: Participants desire to reduce the number of medications they are taking.

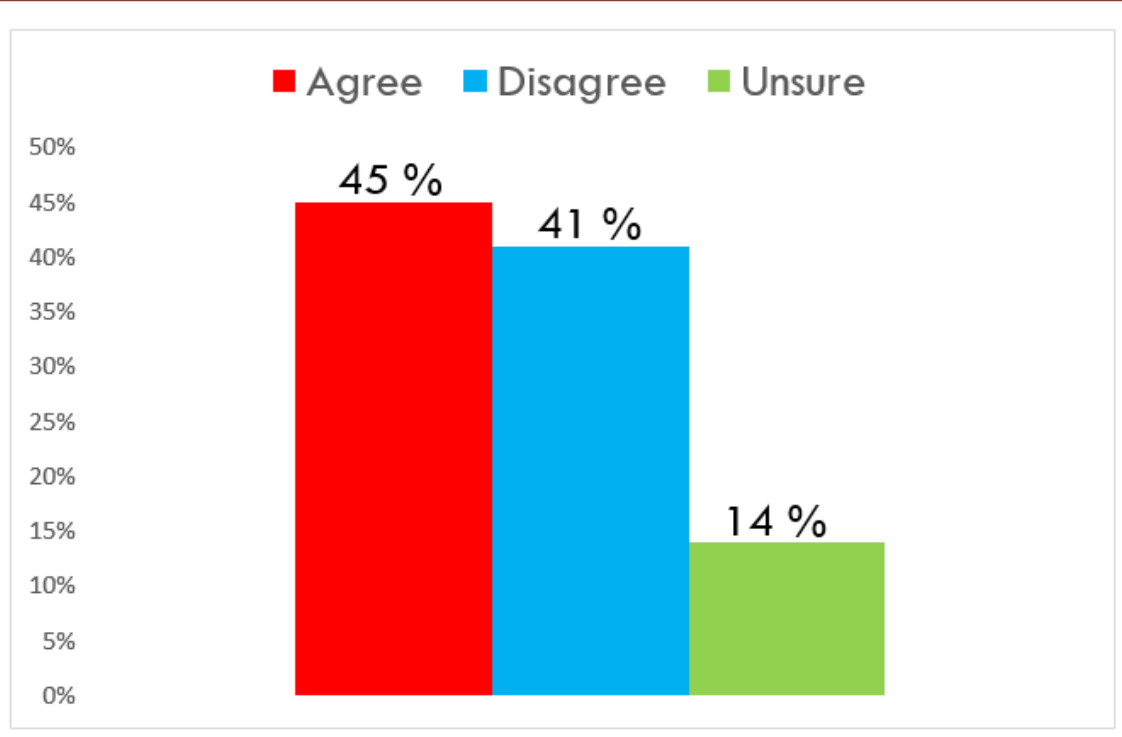

Figure 4: Participants acceptance of taking more medications for their health condition

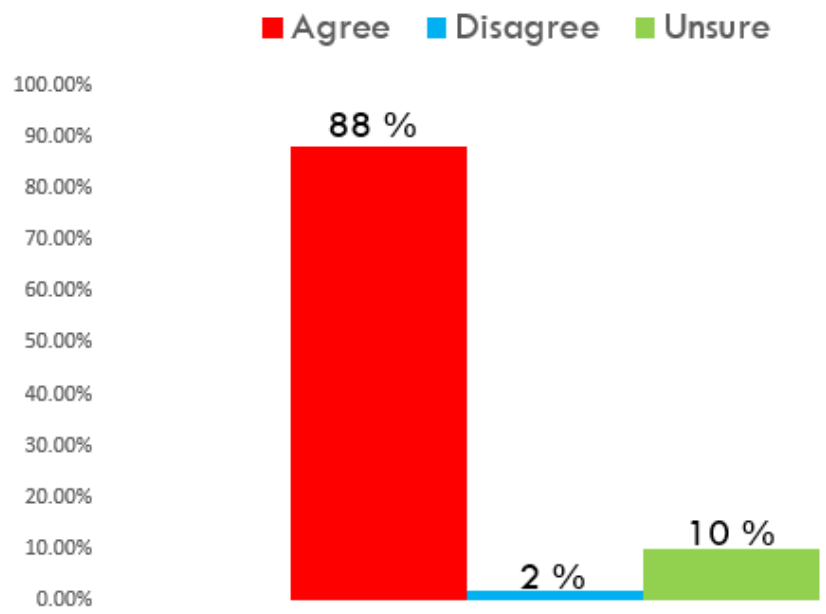

Figure 5: Participants' knowledge of the reasons they were prescribed each of their medications. 
- Agree Disagree $\square$ Unsure

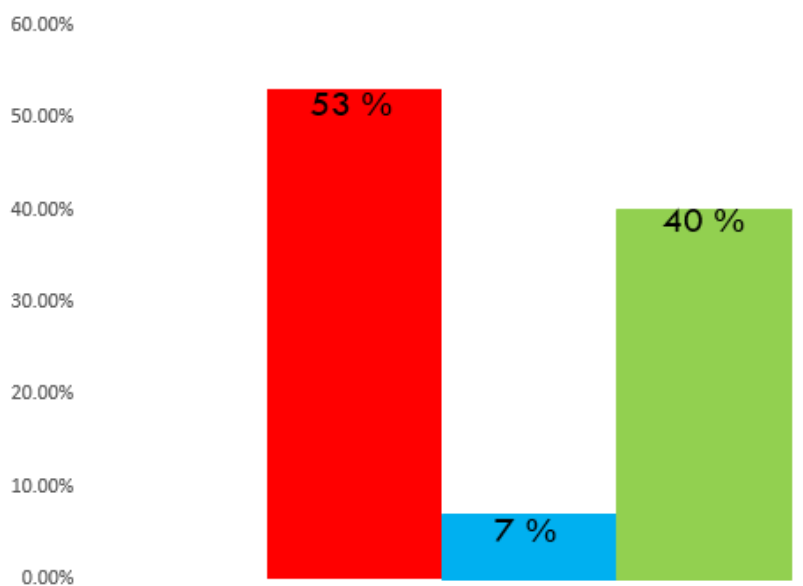

Figure 6: Participants belief in that one or more of their medications causing them side effects.

The results showed that more than $54 \%$ of the participants had never tried to stop taking their medications. On the other hand, approximately $46 \%$ of the participants were noncompliant (Figure 7).

The intake of any number of tablets or capsules was found to be acceptable by $50 \%$ of participants, while $26 \%$ preferred 2 to
5 tablets or capsules per day, and $23 \%$ preferred 6 to 10 tablets or capsules per day (Figure 8). The use of herbal products or nutritional supplements was found to be popular in $34 \%$ of participants as they admitted taking one or two of these. However, $66 \%$ of the participants denied using any herbal products or nutritional supplements (Figure 9).

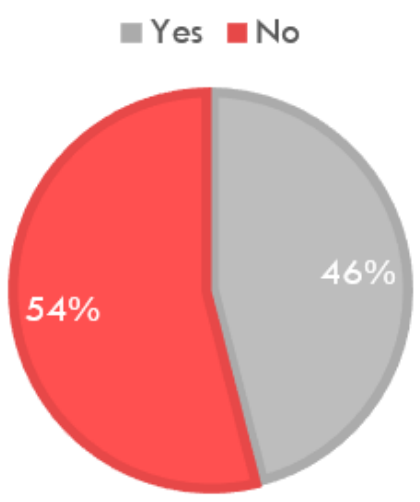

Figure 7: Participants who tried to stop a regular medication.

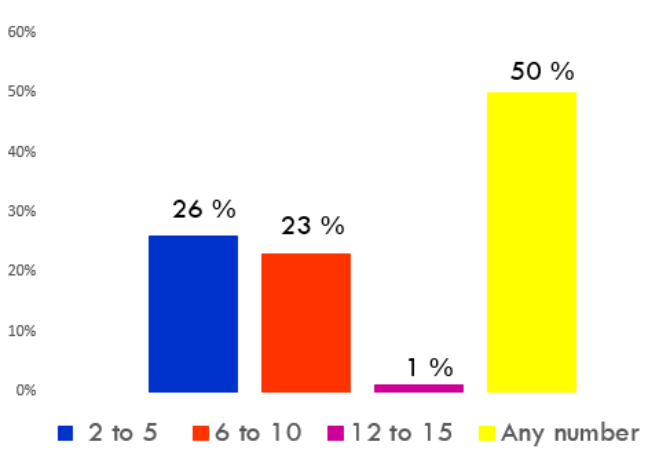

Figure 8: The maximum number of tablets or capsules the participants would be comfortable taking per day. 


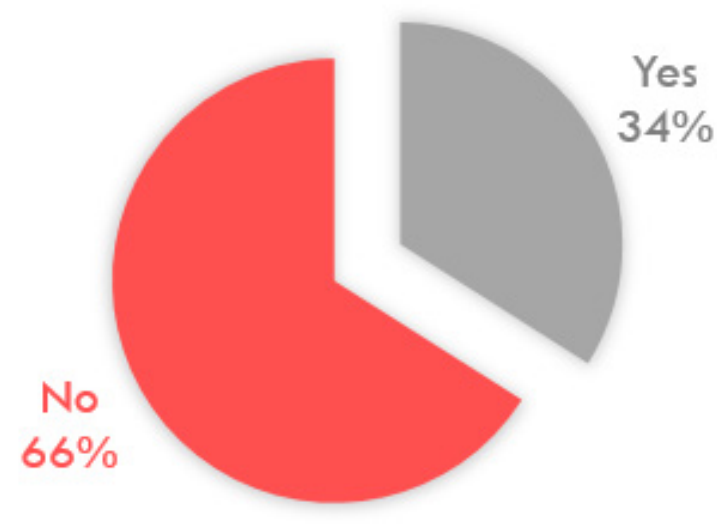

Figure 9: The use of herbal products or nutritional supplements by participants.

Participants gain most of their knowledge about drugs from physicians (100\%) and pharmacists (50\%). Only $10 \%$ of participants read the medication leaflet before using the drug.
Other sources were found to be family and friends, internet and media (Figure 10).

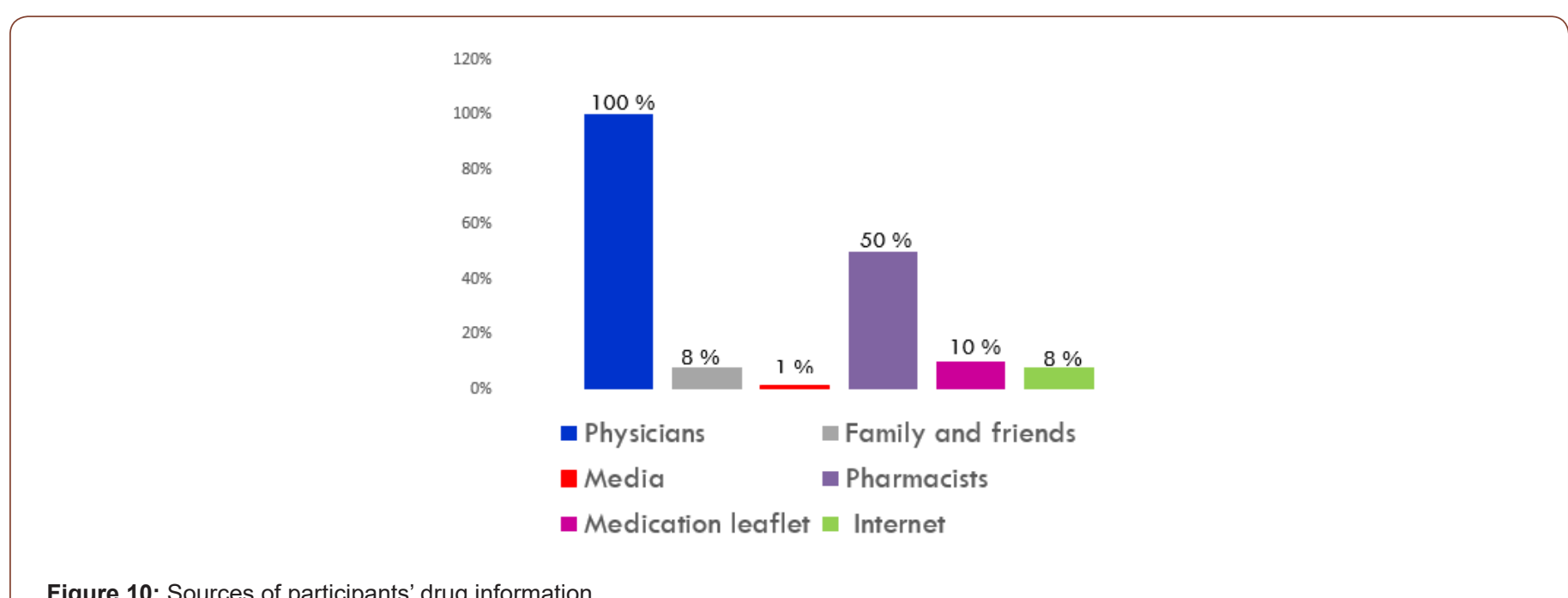

Figure 10: Sources of participants' drug information.

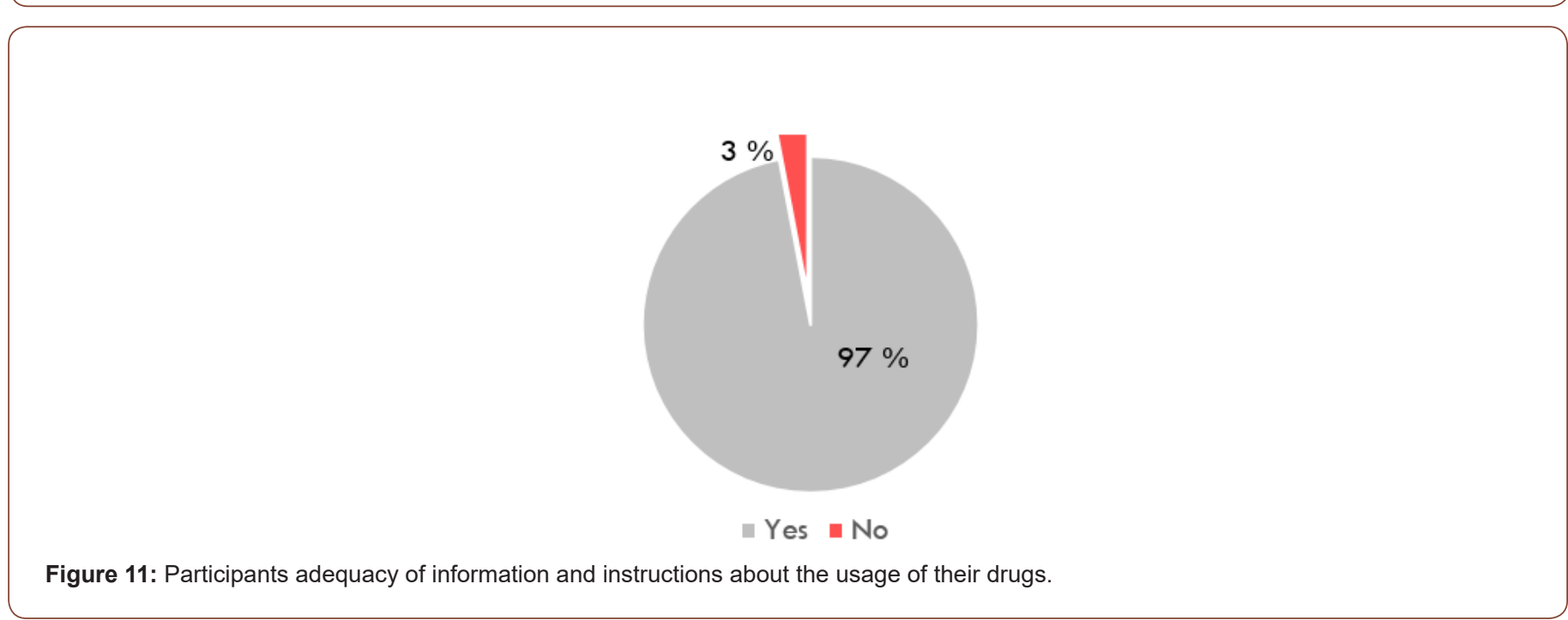


The majority (97\%) of participants claimed that they have enough information and instructions about the usage of their drugs. However, only 3\% admitted lack of adequate knowledge and instructions (Figure 11). Although 95\% of respondents thought that their medications helped them to get well, $5 \%$ thought differently (Figure 12). It was observed that $67 \%$ of the participants did not need admission to hospital in the last year; while $24 \%$ were hospitalized at least once (Figure 13).

The results showed that $56 \%$ of the participants reported that they would be comfortable if their physician stopped one or more of their regular medication, while $25 \%$ feels that all their medications are necessary, and they would be uncomfortable to stop any of them (Figure 14).

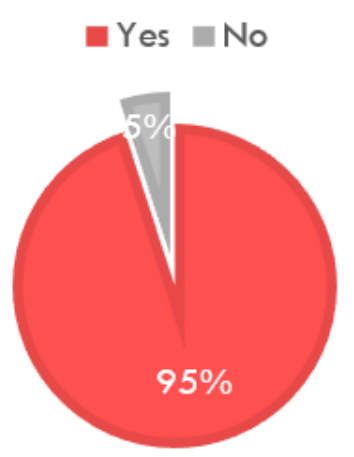

Figure 12: Participants belief in their medications.

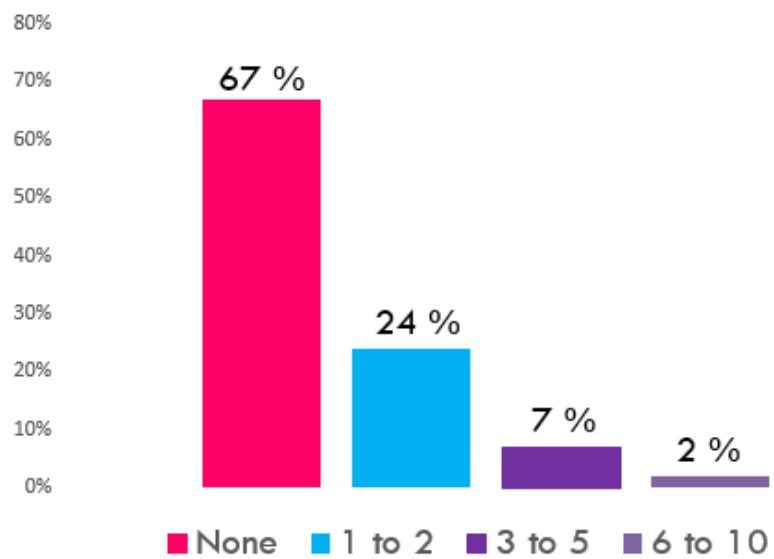

Figure 13: The number of hospitalizations of patients during the previous year.

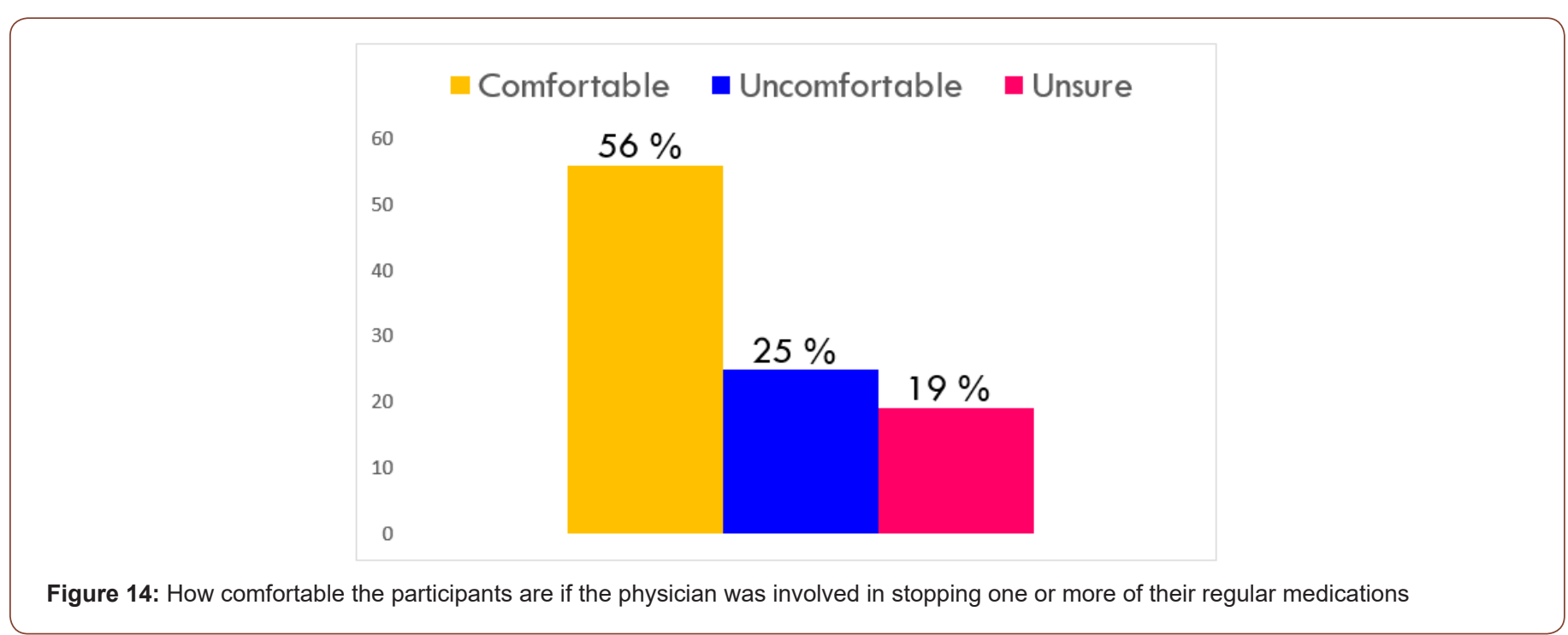




\section{Discussion}

This study shows that the elderly use of prescribed drugs and the mean number of medications being used is growing. This would be justified partially to be appropriate and attributed to the advanced age and its accompanied pathophysiological [6-8]. Moreover, the findings indicate that the pattern of drug consumption among the older adults living in Bahrain was not satisfactory as the average number of medications consumed by the participants in this study was $8.14 \pm 1.22$. However, this is comparable to similar studies such as that in Austria which reported the mean number of drugs taken to be $7.5 \pm 3.8$ [20]. The polypharmacy was found to be common among elderly community and is directly related to the age and the availability of multiple health problems, but inversely to the educational level. Similarly, Golchin, et al. mentioned that the polypharmacy is not related to age and education level which may be due to the small sample size [21]. Another reason for the polypharmacy in the current study could be that the elderly patients tend to visit multiple health care providers which was reported in the literature $[6,11]$. The findings of the current study are comparable to that reported by [22]. The mentioned study has shown that the polypharmacy among older adults in Tehran population is similar to that in Bahrain with small insignificant difference in regard to the correlation between polypharmacy and socio-demographic characteristics and polypharmacy prevalence for prescribed drugs and non-prescribed drugs [22]. The spread of the problem of inappropriate polypharmacy is wide and even "Hyper polypharmacy" was reported in $23.8 \%$ of an Australian study sample [23]. Hyper polypharmacy was defined in this study as the use of more than 10 medication [23].

Additionally, the current study reflects the dilemma that people face between understanding the need to take medications to maintain quality of life and control their health and a general dislike of taking medications. The results showed that the majority of participants desire to reduce the number of medications they are taking especially with the belief that at least one of the medications used is causing them adverse drug reaction. Nevertheless, the majority of patients were found to be compliant with their medication as they never tried to stop any of their medications.

Table 2: Basic questions for medication review. Taken from $[11,16]$.

\begin{tabular}{|c|c|}
\hline 1 & Has a full medication history been collected? \\
\hline 2 & How long ago has the medications been reviewed? \\
\hline 3 & Are all drugs taken by the patient indicated and likely to be effective? \\
\hline 4 & Is the patient taking any inappropriate or incompatible medication? \\
\hline 5 & Are there drug(s) that could be discontinued? Are there essential drugs that are not actually prescribed? \\
\hline 7 & Are the dosage, dose frequency and formulation appropriate? \\
\hline 8 & Is the patient at high risk of therapeutic competition? \\
\hline 9 & Are drug interactions present? \\
\hline
\end{tabular}

Furthermore, the use of food supplements / herbs was found to be common in the Bahraini community as $34 \%$ of patients admitted using, which is comparable to that reported in the literature $[6,8]$. The reason for this could be psychosocial factors.

Patients in the current study believe that the information and instructions they have about the usage of their medications is adequate. However, this argument is doubtful to some extent since the sources mentioned are multiple and included proper sources such as physicians and pharmacists (majority), but some are lacking credibility and accuracy such as the family, friends, media and internet. Inadequate patient's knowledge of his health condition and medications is a major factor associated with inappropriate polypharmacy [12]. This problem needs to address carefully and properly in all patients as it is their right to get proper and sufficient medication counselling from its proper channels which are the healthcare workers especially the pharmacists. A further step would be through the pharmacist providing full pharmaceutical care aiming at improving the patients' quality of life [24].

A strategy to control the problem of polypharmacy need to be considered and might be via the implementation of patients' medication reviewing process [25]. Regular medication review needs to be done and a special form / card to be filled for every patient by physician, pharmacist or nurse in each visit with an appropriate diagnosis to be recorded for each medication prescribed. Basic questions need to be asked regarding the drugs used when filling this patients' medication review (Table 2) [11,16]. In patients' medication review, always consider "De-Prescribing" [26]. Evidence of efficacy of the "De-Prescribing" process was reported in many randomized trials and observational studies [26]. A de-prescribing protocol comprising of 5 steps to assess the current and future medications' benefits and harms (Table 3) [26]. Additionally, raising awareness of physicians, pharmacists and patients is crucial [25]. Counselling and education for the patients and their family should always be practiced. Something that needs to be emphasized upon is that medications includes OTCs, herbal products, food supplements as these are associated with high incidence of drug - drug interactions [25]. 
Table 3: Steps of the "De-Prescribing" protocol. Taken from [26]

\begin{tabular}{|c|c|}
\hline 1 & Ascertain all drugs the patient currently taking and the reasons for each one \\
\hline 2 & Consider overall risk of drug-induced harm in individual patients in determining the required intensity of de-prescribing intervention \\
\hline 3 & Assess each drug in regard to its current or future benefit potential compared with current or future harm or burden potential \\
\hline 4 & $\begin{array}{c}\text { Prioritize drugs for discontinuation that have the lowest benefit-harm ratio and lowest likelihood of adverse withdrawal reactions or disease } \\
\text { rebound syndromes }\end{array}$ \\
\hline 5 & Implement a discontinuation regimen and monitor patients closely for improvement in outcomes or onset of adverse effects. \\
\hline
\end{tabular}

A limitation of the current study is the lack of measurement of the incidence of OTC medications use although previous studies reported wide use of OTC analgesics and laxatives among Bahraini patients $[27,28]$.

\section{Conclusion}

The problem of inappropriate polypharmacy is common in Bahrain as it is around the world with the advancement in healthcare services \& growing elderly population. Strategies to control the problem of polypharmacy must always be considered as the research showed that reduction of long-term drugs helps in reducing ADRs and mortality. Other benefits reported are lower incidence of acute hospital admission, reduction of costs and higher levels of patient satisfaction and drug compliance.

\section{Conflict of Interest}

None.

\section{Acknowledgement}

Thanks to Nawal S Makki from the English department at the College of Health \& Sports Sciences for the English language proofreading.

\section{References}

1. Junius Walker U, Theile G, \& Hummers-Pradier E (2007) Prevalence and predictors of polypharmacy among older primary care patients in Germany. [Multicenter Study Randomized Controlled Trial Research Support, Non-U.S. Gov't]. Fam Pract 24(1): 14-19.

2. Koper D, Kamenski G, Flamm M, Bohmdorfer B, Sonnichsen A (2013) Frequency of medication errors in primary care patients with polypharmacy. [Research Support, Non-U.S. Gov't]. Fam Pract 30(3): 313-319.

3. Monégat M, Sermet C, Perronnin M, Rococo E (2014) Polypharmacy: Definitions, Measurement and Stakes Involved Review of the Literature and Measurement Tests. Issues in Health Economics p. 12.

4. Schaefer K, Maerkedahl H, Birk HO, Henriksen LO (2010) Polypharmacy in general practice. Dan Med Bull 57(7): A4165.

5. LeBlanc TW, McNeil MJ, Kamal AH, Currow DC, Abernethy AP (2015) Polypharmacy in patients with advanced cancer and the role of medication discontinuation. [Review]. Lancet Oncol 16(7): e333-341.

6. Austin RP (2006) Polypharmacy as a Risk Factor in the Treatment of Type 2 Diabetes. Diabetes Spectrum 19(1): 13-16.

7. Budnitz DS, Lovegrove MC, Shehab N, Richards CL (2011) Emergency hospitalizations for adverse drug events in older Americans. N Engl J Med 365(21): 2002-2012.

8. Loffler C, Drewelow E, Paschka SD, Frankenstein M, Eger J, et al. (2014) Optimizing polypharmacy among elderly hospital patients with chronic diseases--study protocol of the cluster randomized controlled Polite-Rct trial. [Multicenter Study Randomized Controlled Trial Research Support, Non-U.S. Gov't]. Implement Sci 9: 151.

9. Nobili A, Garattini S, Mannucci PM (2011) Multiple diseases and polypharmacy in the elderly: challenges for the internist of the third millennium. Journal of Comorbidity 1: 28-44.

10. Corsonello A, Abbatecola AM, Fusco S, Luciani F, Marino A, et al. (2015) The impact of drug interactions and polypharmacy on antimicrobial therapy in the elderly. [Review]. Clin Microbiol Infect 21(1): 20-26.

11. Planton J, Edlund BJ (2010) Strategies for reducing polypharmacy in older adults. [Case Reports]. J Gerontol Nurs 36(1): 8-12.

12. Stawicki SP, Gerlach AT (2009) Polypharmacy and medication errors: Stop, Listen, Look, and Analyze.... OPUS 12 Scientist 3(1): 6-10.

13. Fried TR, O'Leary J, Towle V, Goldstein MK, Trentalange M, et al. (2014) Health outcomes associated with polypharmacy in community-dwelling older adults: a systematic review. [Research Support, NIH, Extramural Research Support, Non-U.S. Gov't Review]. J Am Geriatr Soc 62(12): 2261-2272.

14. Hajjar ER, Cafiero AC, Hanlon JT (2007) Polypharmacy in elderly patients. [Review]. Am J Geriatr Pharmacother 5(4): 345-351.

15. Hilmer SN, Gnjidic D (2009) The effects of polypharmacy in older adults. [Research Support, Non-U.S. Gov't]. Clin Pharmacol Ther 85(1): 86-88.

16. Mannucci PM, Nobili A (2014) Multimorbidity and polypharmacy in the elderly: lessons from REPOSI. [Review]. Intern Emerg Med 9(7): 723734.

17. Sganga F, Landi F, Ruggiero C, Corsonello A, Vetrano DL, et al. (2015) Polypharmacy and health outcomes among older adults discharged from hospital: results from the CRIME study. [Research Support, NonU.S. Gov't]. Geriatr Gerontol Int 15(2): 141-146.

18. Ramanath KV, Nedumballi S (2012) Assessment of Medication-Related Problems in Geriatric Patients of a Rural Tertiary Care Hospital. Journal of Young Pharmacists: JYP 4(4): 273-278.

19. Shalini, Joshi MC (2012) Study of polypharmacy and associated problems among elderly patients. Internet Journal of Medical Update January 7(1): 35-39.

20. Schuler J, Duckelmann C, Beindl W, Prinz E, Michalski T, et al. (2008) Polypharmacy and inappropriate prescribing in elderly internalmedicine patients in Austria. [Research Support, Non-U.S. Gov't]. Wien Klin Wochenschr 120(23-24): 733-741.

21. Golchin N, Frank S, Vince A, Isham L, Meropol S (2015) Polypharmacy in the elderly. [Brief Communication]. Journal of Research in Pharmacy Practice 4(2): 85-88.

22. Ahmadi B, Alimohamadian M, Mahmoodi M (2006) Polypharmacy among older adults in Tehran. Tehran University Medical Journal 64(9): 65-71.

23. Hubbard RE, Peel NM, Scott IA, Martin JH, Smith A, et al. (2015) Polypharmacy among inpatients aged 70 years or older in Australia. [Observational Study]. Med J Aust 202(7): 373-377.

24. Swamy K, Ramani JU, Ramesh B, Radadiya M, Sowmya B, Patel D (2014) Prevalence of Polypharmacy and Drug to Drug Interactions in A Tertiary Care Teaching Hospital. International Research Journal of Pharmacy 5(10): 778-782. 
25. Rambhade S, Chakarborty A, Shrivastava A, Patil UK, Rambhade A (2012) A Survey on Polypharmacy and Use of Inappropriate Medications. Toxicology International 19(1): 68-73.

26. Scott IA, Hilmer SN, Reeve E, Potter K, Le Couteur D, et al. (2015) Reducing inappropriate polypharmacy: the process of deprescribing [Research Support, Non-U.S. Gov't]. JAMA Intern Med 175(5): 827-834.
27. Al Qallaf SM (2015) Evaluating Knowledge of Over the Counter Analgesics Among Bahraini People. Asian J Pharm Clin Res 8(3): 266-270.

28. Al Qallaf SM, Aqeel D (2016) Analgesics and Laxatives Abuse and Associated Habits in Bahrain: Exploratory Study. International Journal of Institutional Pharmacy and Life Sciences 6(2): 1-9. 